\title{
Efeito da Presença de Defeitos no Comportamento Mecânico de J untas Coladas Carbono-Epoxi
}

\author{
Nara G. Berry e J osé Roberto M. d'Almeida \\ Departamento de Ciência dos Materiais e Metalurgia, PUC - Rio
}

\begin{abstract}
Resumo: Juntas unidas por adesão vêm sendo largamente empregadas em substituição a peças unidas por métodos convencionais de fixação mecânica. A confiabilidade destas juntas deve considerar o efeito da presença de defeitos na área colada sobre o comportamento mecânico da peça. Neste trabalho analisou-se o comportamento mecânico de juntas sobrepostas simples em função da presença de defeitos. Foi verificado, também, a adequação para as juntas com defeitos dos principais modelos teóricos desenvolvidos para juntas sem defeitos.
\end{abstract}

Palavras-chave: Compósitos, juntas sobrepostas simples, defeitos, comportamento mecânico.

\section{Effect of Defects on the Mechanical Behavior of Carbon Fiber-Epoxy Matrix Composites Single-Lap J oints}

Abstract: Nowadays, bonded joints are been widely used to replace mechanically joined parts. The influence of defects on the mechanical behavior of these joints must be determined if their reliability is to be ascertained. In this work, the mechanical behavior of single lap joints was evaluated as a function of the presence and size of defects on the bonded area. The results obtained indicate that the models developed for determining the strength of flawless single lap joints can be employed to model the behavior of single lap joints with defects.

Keywords: Composites, single lap joints, defects.

\section{Introdução}

O uso de adesivos para a união de materiais tem se tornado uma prática corrente nos mais diversos ramos da engenharia. A técnica de adesão é particularmente interessante quando se quer unir materiais diferentes ou com baixa resistência à temperatura, onde o emprego de uma técnica convencional de união, tal como soldagem, apresenta diversas desvantagens. Assim sendo, a técnica de adesão está bastante difundida nas indústrias aeroespacial (em painéis e peças em compósitos) e naval ${ }^{[1]}$, bem como em aplicações não estruturais de engenharia tal como na aplicação de filmes protetores sobre peças fabricadas em plástico para a indústria automobilística ${ }^{[2]}$.

A confiabilidade de uma junta unida por adesão depende tanto de fatores intrínsecos à junta, tal como a sua geometria, como extrínsecos, tais como as condições operacionais às quais a junta é submetida. Além destes fatores, a confiabilidade de uma junta depende também da presença de defeitos, que podem ou não ser admissíveis. Constatada a presença de um defeito, deve-se verificar sua influência sobre o comportamento da junta e determinar qual tamanho de defeito determina que o membro estrutural precisará ser substituído ou reparado.

O objetivo deste trabalho foi avaliar o desempenho de juntas do tipo sobreposta simples, de compósito de fibra de carbono e matriz epoxi, em função do tamanho do defeito. Um adesivo epoxi foi utilizado para a união da junta e os defeitos foram simulados empregando-se discos de Teflon. O emprego de Teflon com esta finalidade é bastante eficiente, pois não há qualquer ligação entre o defeito e o adesivo empregado ${ }^{[3]}$.

Autor para correspondência: José Roberto M. d'Almeida, Departamento de Ciência dos Materiais e Metalurgia, Pontifícia Universidade Católica do Rio de Janeiro, Rua Marquês de São Vicente, 225, Gávea, CEP: 22453-900, Rio de Janeiro, RJ. E-mail: dalmeida@dcmm.puc-rio.br 


\section{Aspectos Teóricos}

Assegurando-se que as juntas estejam cuidadosamente projetadas para se adaptarem às condições operacionais do adesivo, as estruturas coladas freqüentemente provam ser mais seguras do que aquelas montadas usando processos convencionais de fixação mecânica ${ }^{[1]}$. Contudo, deve ser levado em conta que uma simples troca do processo de fixação mecânica pelo processo de união por adesivos é raramente bem sucedida ${ }_{2}$ especialmente quando é exigida elevada resistência do adesivo. Em particular, dois fundamentos devem ser observados ${ }^{[1]}$ : (1) maximizar a área de trabalho colada e (2) empregar uma geometria favorável no projeto da junta.

Juntas sobrepostas têm sido bastante estudadas e são as mais comumente usadas ${ }^{[4]}$. Na junta sobreposta simples duas placas formam um sobreposto e são unidas por uma camada de adesivo, conforme mostrado na Figura 1. O carregamento no plano das placas, normalmente chamadas de aderentes ou substratos, produz tensões cisalhantes e tensões normais no adesivo e nas faces coladas das placas ${ }^{[5]}$. A junta pode falhar tanto no adesivo quanto na interface ou nos aderentes, de acordo com as tensões desenvolvidas em cada uma destas partes e, também, de acordo com a resistência do adesivo, da interface e dos aderentes. A adesão promovida pelos adesivos modernos é, normalmente, alta, e a ruptura freqüentemente ocorre no adesivo, como por exemplo em juntas metal-metal, ou nos aderentes, como acontece com juntas de madeira ${ }^{[6]}$.

As tensões desenvolvidas nas juntas sobrepostas simples não são uniformes e a tensão cisalhante máxima, $\tau_{\text {máx }}$, pode ser muitas vezes superior a tensão média, $\tau_{\mathrm{m}}$, que é definida como a razão entre a carga aplicada, $\mathrm{P}$, e a área real de colagem. A relação entre estas tensões é dada por $\tau_{\text {máx }}=\eta \cdot \tau_{\mathrm{m}}$, onde $\eta$ é um fator de concentração de tensões, que depende de parâmetros geométricos da junta e das constantes elásticas dos materiais que a constituem ${ }^{[7]}$.

A existência de uma deformação diferencial nestas juntas pode ser compreendida pela comparação entre uma junta formada por aderentes inextensíveis e outra com membros rígidos, ou seja, extensíveis

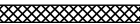

Figura 1. Junta do tipo sobreposta simples.

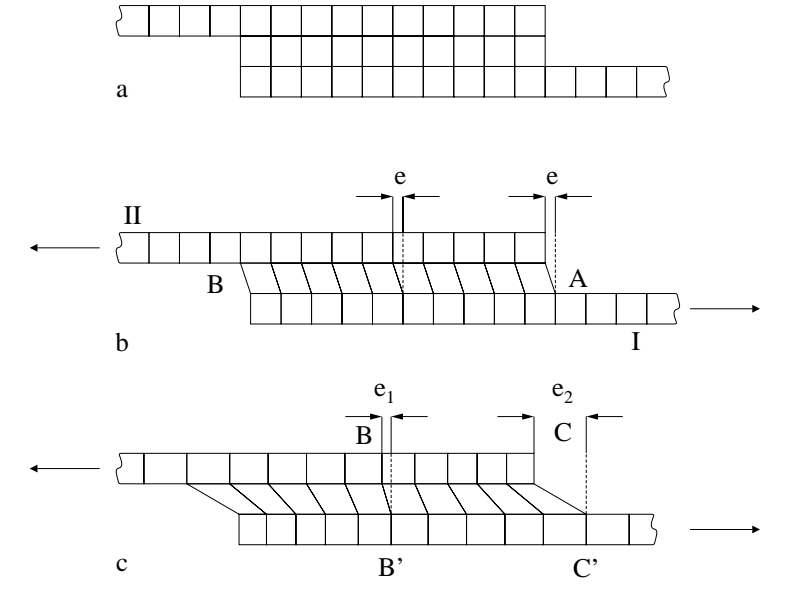

Figura 2. Representação esquemática do cisalhamento no adesivo em uma junta sobreposta: (a) antes do carregamento, (b) aderentes inextensíveis, (c) aderentes extensíveis.

porém que não defletem. Ao se carregar a junta, Figura 2a, os aderentes inextensíveis irão se deslocar como blocos sólidos e o adesivo sofrerá uma deformação cisalhante, deformando-se de forma a acomodar o deslocamento, e, dos aderentes, que será o mesmo em toda a junta, Figura 2b. Porém, cada aderente suporta toda a carga aplicada até imediatamente antes do sobreposto e a transmite gradualmente através do adesivo. Deste modo, a tensão no membro I será maior no ponto A e diminuirá gradualmente em direção ao ponto $\mathrm{B}$, onde será nula, Figura $2 \mathrm{~b}$. Contrariamente, a tensão do aderente II será maior em $\mathrm{B}$ e será nula em A.

Esta variação de tensão cisalhante não será importante se os aderentes I e II se comportarem como sólidos inextensíveis, mas se eles forem extensíveis desenvolverão deformações proporcionais às tensões existentes. A distribuição de deformações será como mostrado na Figura 2c, onde os pontos B-B', perto da junta, e C-C', nas extremidades do sobreposto, estarão sujeitos a deslocamentos diferentes $\mathrm{e}_{1} \mathrm{e} \mathrm{e}_{2}$, respectivamente. Pode ser observado que o deslocamento da extremidade do sobreposto, $\mathrm{e}_{2}$, é bem maior, o que resulta numa tensão cisalhante maior no adesivo nas extremidades do sobreposto. Resultados experimentais mostram que, de fato, a fratura de uma junta deste tipo começa, preferencialmente, na borda da junta $^{[7,8]}$.

Os aderentes de uma junta sobreposta estão, necessariamente, deslocados axialmente entre si por, pelo menos, sua espessura, Figura 3a. Assim, a linha de carregamento que une os pontos de aplicação da carga P será oblíqua e passará pelo ponto médio da jun- 

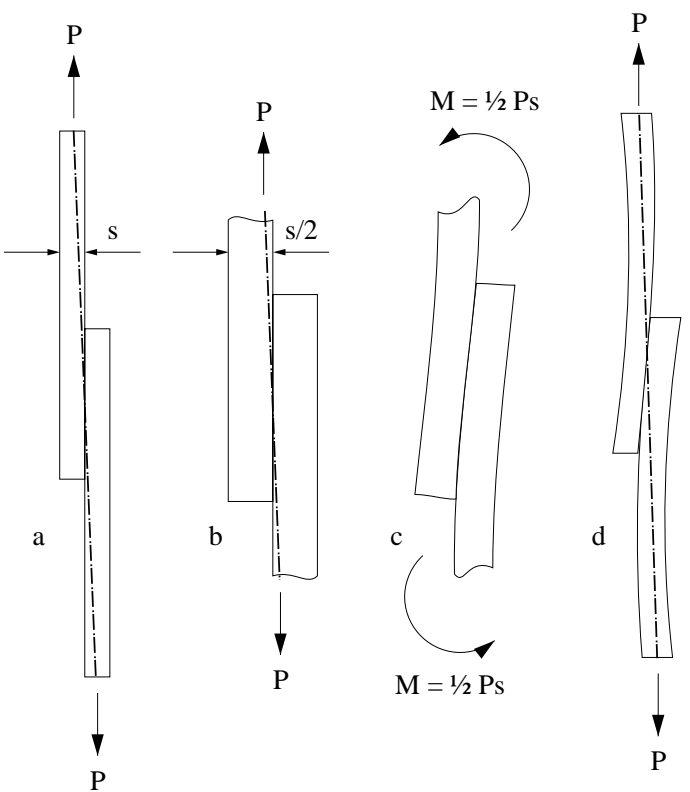

Figura 3. Representação esquemática da flexão dos aderentes e das tensões normais resultantes nas extremidades do sobreposto: (a) junta sobreposta antes da deformação, (b) excentricidade da carga, (c) momento fletor, (d) deformação final da junta.

ta. Se o comprimento de cada membro fora do sobreposto for bem maior que o sobreposto, a linha de carregamento passará ao longo das faces internas de cada membro, Figura 3b, imediatamente antes do sobreposto. A excentricidade de carga gera tensões normais à superfície da junta. Estas tensões estão, entretanto, confinadas às regiões adjacentes às extremidades do sobreposto, mas podem reduzir consideravelmente a resistência destas juntas ${ }^{[5,7]}$.

A excentricidade da carga em cada aderente causará um momento fletor, $\mathrm{M}=1 / 2 \mathrm{P}$. $\mathrm{s}$, onde $\mathrm{P}$ é a carga aplicada e s a espessura do aderente, Figura 3c. Sob a ação deste momento fletor, os aderentes, caso sejam suficientemente longos, fletem e a junta fica deformada, como mostra a Figura 3d. Esta deformação reduz as tensões normais, pois a linha de carregamento fica mais próxima do eixo central de cada membro, reduzindo o momento fletor. A junta ao se deformar, assume uma posição com quantidade mínima de energia de deformação ${ }^{[7]}$.

\section{Modelos para Análise da Distribuição de Tensões em J untas Sobrepostas}

Diversos modelos têm sido desenvolvidos para analisar a distribuição de tensões em juntas sobrepostas. Aqueles que são mais empregados serão apresentados neste trabalho.

\section{A Análise de Volkersen ${ }^{[9]}$}

Esta análise considera apenas as tensões cisalhantes devido à deformação diferencial, desprezando as tensões normais devidas à flexão dos aderentes. $\mathrm{O}$ modelo considera que os aderentes estão submetidos a um carregamento trativo uniaxial e as tensões cisalhantes são analisadas apenas na camada do adesivo, que tem espessura uniforme e cujas extremidades são planas e normais à direção de aplicação da carga. Para materiais elásticos o modelo mostra que ${ }^{[7]}$ :

$$
\eta=\tau_{\max } / \tau_{\mathrm{m}}=\sqrt{\Delta / W} \frac{W-1+\cosh \Delta W}{\operatorname{senh} \sqrt{\Delta . W}}
$$

Onde $\eta$ é o fator de concentração de tensões, $\tau_{\max }$ é a tensão de cisalhamento máxima e $\tau_{\mathrm{m}}$ é a tensão de cisalhamento média.

Os parâmetros $\Delta \mathrm{e}$ W são dados pelas equações:

$$
\Delta=\frac{G \cdot L^{2}}{E_{2} \cdot s_{2} \cdot d}
$$

e

$$
W=\left(\frac{E_{1} \cdot s_{1}+E_{2} \cdot s_{2}}{E_{1} \cdot s_{1}}\right)
$$

onde $\mathrm{E}_{1}$ e $\mathrm{E}_{2}$ são os módulos de elasticidade e $\mathrm{s}_{1}, \mathrm{~s}_{2}$ as espessuras dos aderentes; $G$ é o módulo de cisalhamento do adesivo, d é a espessura da camada de adesivo e L é o comprimento do sobreposto.

\section{Modelo de Goland e Reissner ${ }^{[10]}$}

Neste modelo a deformação dos aderentes fora do sobreposto é caracterizada por um parâmetro, $\mathrm{k}$, que é a razão entre o momento fletor atuando imediatamente antes do sobreposto e o valor deste momento para membros inflexíveis. $\mathrm{O}$ valor de $\mathrm{k}$ é determinado pela equação:

$$
\frac{1}{\mathrm{k}}=1+2 \sqrt{2} \tanh \left\{\sqrt{\frac{3}{2}} \sqrt{\left(1-v^{2}\right)} \frac{L}{2 s} \cdot \sqrt{\frac{\sigma}{E}}\right\}
$$

onde E e $v$ são os módulos de elasticidade e o coeficiente de Poisson dos aderentes, L é o comprimento do sobreposto, s é a espessura do aderente e $\sigma$ é a tensão aplicada nas extremidades do aderente e longe da área sobreposta. 
Pode-se constatar que o fator adimensional $\mathrm{k}$ depende da geometria da junta ( $\mathrm{L} \mathrm{e} \mathrm{s}$ ), das propriedades elásticas dos aderentes ( $\mathrm{E}$ e $\mathrm{v}$ ) e ainda da tensão remota nos aderentes $(\sigma)$. Portanto, $\mathrm{k}$ varia com a carga externa aplicada. $\mathrm{O}$ valor de $\mathrm{k}$ será igual a 1 para aderentes indeformáveis, ou por causa de sua rigidez ou porque a carga aplicada é pequena. Para materiais menos rígidos, ou um valor alto de carga, $\mathrm{k}$ diminui. Na prática este parâmetro permanece normalmente acima de $0,35^{[7]}$.

Sendo $\mathrm{E}_{\mathrm{a}} \mathrm{o}$ módulo de elasticidade do adesivo e conhecendo-se a deformação dos aderentes e a tensão remota, $\sigma$, a qual é dada pela razão entre a carga aplicada e a área da seção resistente do substrato, o cálculo da distribuição de tensões na junta é considerado como um problema de deformação plana para dois casos extremos ${ }^{[10]}$ : (1) as camadas de adesivo são muito finas e rígidas para influírem na flexibilidade da junta. Esta condição é satisfeita se $\frac{d}{E_{a}}>\frac{s}{E}$; (2) camadas de adesivo flexíveis. Esta condição é satisfeita se $\frac{d}{E_{a}}<\frac{s}{E}$.

Quando a camada de adesivo for considerada muito fina ela passa a ser desprezada e a junta, como um todo, é considerada como um material homogêneo e contínuo $^{[10]}$. As tensões calculadas são aquelas nos aderentes e, ao longo da linha de colagem, elas podem ser consideradas iguais àquelas atuantes na camada de adesivo.

Em juntas com camadas de adesivo relativamente flexíveis, tanto a tensão cisalhante quanto a tensão normal têm seus valores máximos na borda do sobreposto. Neste modelo o fator de concentração de tensões, $\eta$, pode ser expresso como ${ }^{[10]}$.

$$
\eta=\frac{1+3 k}{4} \cdot \sqrt{2 \Delta} \cdot \operatorname{cotanh} \sqrt{2 \Delta}+\frac{3}{4}(1-k)
$$

A equação (5) é obtida da equação (4) pela introdução da tensão cisalhante ao invés da tensão remota, $\sigma$, e pelo fator $\Delta$ dado pela equação (2). Pode-se observar que $\eta=\mathrm{f}(\Delta, \mathrm{k})$ onde, com a introdução do fator $\mathrm{k}$, os efeitos de flexão passam a ser considerados.

Conhecendo-se o valor de $\eta$, a máxima tensão normal, $\sigma_{\text {máx }}$, atuando na borda do sobreposto pode ser determinada. As equações desenvolvidas por Goland e Reissner não são entretanto de fácil emprego e foram simplificadas por Mylonas ${ }^{[11]}$ que mostrou que:

$$
\sigma_{\text {máx }}=\eta_{1} \cdot \sigma
$$

onde $\eta_{1} \cong \frac{1}{2} \cdot k \cdot \gamma^{2}$. Na equação (6) $\sigma$ é a tensão remota aplicada à junta e $\gamma$ é um fator dependente apenas das flexibilidades relativas dos aderentes e da camada de adesivo e é dado pela equação:

$$
\gamma^{4}=6 \cdot \frac{E_{a} \cdot s}{E . d}
$$

$O$ fator de concentração para tensões normais, $\eta_{1}$, é função apenas de $\mathrm{k}$ e de $\gamma$, sendo independente do comprimento do sobreposto, L, exceto pela contribuição deste em k [equação (4)]. Ou seja, L influencia a tensão normal apenas por afetar a deformação dos aderentes.

Mylonas propôs também que a máxima tensão normal $\left(\tau_{\max }\right)$ está relacionada com a tensão cisalhante média $\left(\tau_{\mathrm{m}}\right)$ atuante na junta, da seguinte forma ${ }^{[11]}$ :

$$
\sigma_{\text {máx }}=2 . \mathrm{k} \cdot \tau_{\mathrm{m}} \cdot \sqrt{\Delta}
$$

\section{Modelo de Hart-S mith ${ }^{[12]}$}

Hart-Smith ${ }^{[12]}$ considerou que os aderentes e o adesivo se comportam como materiais elasto-plásticos, e não como materiais elásticos, especialmente quando tensões cisalhantes estão atuando. $\mathrm{O}$ modelo proposto foi desenvolvido empregando soluções analíticas fechadas. Este modelo mostra que a inclusão da plasticidade do adesivo, quando este está submetido a carregamento cisalhante, pode reduzir substancialmente a concentração de tensões e, portanto, aumentar a resistência prevista das juntas em comparação com a análise que considera os materiais como sendo puramente elásticos.

No caso de uma junta sobreposta simples com aderentes idênticos, considerando as tensões trativas transversais, $\sigma_{11}$, e assumindo que o adesivo só apresenta comportamento elástico, tem-se:

$$
\sigma_{11(\max )}=M_{e} \cdot\left(\frac{E_{a}}{2 \cdot d \cdot D_{b} \cdot b}\right)^{1 / 2}
$$

onde $\mathrm{D}_{\mathrm{b}}$ é a rigidez à flexão por unidade de largura dos aderentes, a qual é dada pela equação:

$$
D_{b}=\frac{E_{s} \cdot d^{3}}{12 \cdot\left(1-v_{s}^{2}\right) \cdot b}
$$


e $\mathrm{M}_{\mathrm{e}}$ é o momento fletor por unidade de largura dos aderentes nas extremidades do sobreposto. $\mathrm{M}_{\mathrm{e}}$ é calculado pela relação:

$$
M_{e}=k \cdot \frac{P(d+s)}{2 \cdot b}
$$

onde k é dado pela equação (4) e P é a carga aplicada.

A equação (9) fornece o valor máximo de tensão normal no adesivo, $\sigma_{11}$, nas extremidades do sobreposto. Deve-se ressaltar que os resultados finais encontrados por Hart-Smith concordam bastante bem com os da análise feita por Goland e Reisner ${ }^{[7]}$.

\section{Materiais e Métodos Experimentais}

O material utilizado como substrato foi um compósito de fibras unidirecionais de carbono em matriz epoxi, com fração volumétrica de fibras de 0,55 . A especificação deste material e o método empregado em sua fabricação, estão descritos em trabalhos anteriores ${ }^{[13,14]}$. Particularmente importante para este trabalho foi o fato destes compósitos apresentarem uma espessura bastante uniforme $(1,59 \mathrm{~mm})$, com variação menor do que $4 \%$. Assim, foi possível, posteriormente, verificar com precisão a espessura e uniformidade da camada de adesivo aplicada.

A rugosidade dos substratos foi determinada, tendo em vista que este parâmetro influencia de modo importante o espalhamento do adesivo ${ }^{[15]}$. As análises foram feitas em um rugosímetro automatizado e foram medidos 8 perfis de rugosidade, para avaliar a uniformidade do acabamento superficial destes materiais.

Juntas sobrepostas simples, Figura1, foram fabricadas com $80 \mathrm{~mm}$ de comprimento do substrato, $\mathrm{L}, 25,4 \mathrm{~mm}$ de comprimento do sobreposto, $\mathrm{L}_{\mathrm{c}}$, e
25,4 mm de largura da junta, b; de modo que as juntas foram fabricadas com área sobreposta de 25,4 mm x 25,4 mm. Como adesivo foi empregada uma resina epoxi bifuncional de cura a frio, formada pelo monômero epoxi diglicidil-éter do bisfenol-A (DGEBA) e pela amina alifática hexafuncional trietileno tetramina (TETA). A quantidade de endurecedor, TETA, usada foi de 13 partes em peso, para 100 partes do monômero, que corresponde a formulação estequiométrica para este sistema epoxi ${ }^{[16]}$.

Para assegurar que a superfície do substrato estivesse livre de contaminantes foi feita limpeza com uma escova de cerdas macias e, posteriormente, a superfície foi jateada com ar à temperatura ambiente. Nenhum prétratamento químico ou mecânico foi realizado, pois estes não são indicados para este tipo de substrato, já que poderiam, respectivamente, afetar a matriz polimérica ou danificar as fibras do compósito ${ }^{[6]}$.

A técnica empregada para aplicação do adesivo foi a de pincelamento ${ }^{[1]}$. $\mathrm{O}$ adesivo foi aplicado de modo cuidadoso para não promover a formação de bolhas de ar e de modo a se obter uma película com espessura uniforme. A superfície de colagem preparada consistiu de uma área quadrada com 25,4 mm de lado. A espessura da camada de adesivo foi determinada posteriormente à colagem, medindo-se a espessura total das juntas com um micrômetro. Este procedimento pôde ser adotado tendo em vista a uniformidade da espessura dos compósitos usados como substrato.

Para garantir uma distribuição uniforme do adesivo e fabricar juntas com espessura constante, aplicou-se uma pressão de 2,7 kPa sobre cada corpo de prova. Este valor foi determinado experimentalmente, pois os valores médios de pressão indicados pela literatura ${ }^{[17]}$ não forneceram bons resultados quando aplicados diretamente ao sistema aderente-adesivo aqui empregado.

Tabela 1. Especificação das juntas fabricadas e valores experimentais para a carga de ruptura.

\begin{tabular}{cccc|c}
\hline Especificação da junta & $\begin{array}{c}\text { Diâmetro do defeito } \\
(\mathbf{m m})\end{array}$ & \% área com defeito & $\begin{array}{c}\text { Carga de ruptura } \\
(\mathbf{N})\end{array}$ & $\begin{array}{c}\text { Carga de ruptura* } \\
(\mathbf{N})\end{array}$ \\
\hline $\mathrm{D}_{0}$ & 0 & 0 & $6127 \pm 1102$ & $6127 \pm 1102$ \\
$\mathrm{D}_{4}$ & 4 & 2 & $4302 \pm 701$ & \\
$\mathrm{D}_{6}$ & 6 & 4,4 & $4851 \pm 408$ & $4618 \pm 554$ \\
$\mathrm{D}_{7}$ & 7 & 6 & $4655 \pm 1240$ & \\
$\mathrm{D}_{11}$ & 11 & 15 & $5194 \pm 655$ & \\
$\mathrm{D}_{16}$ & 16 & 30 & $3405 \pm 557$ & $3405 \pm 557$ \\
$\mathrm{D}_{20}$ & 20 & 50 & $2795 \pm 359$ & $2795 \pm 359$ \\
\hline
\end{tabular}

* valores estatisticamente corrigidos 
Os defeitos foram introduzidos colocando-se no centro da junta um disco de Teflon, uma vez que este material não adere à resina epoxi ${ }^{[3,4]}$. $\mathrm{O}$ diâmetro dos defeitos variou entre 4 e $20 \mathrm{~mm}$, correspondendo a uma variação entre 2 e $50 \%$ de área colada, Tabela 1.

Para determinação da carga de ruptura, as juntas foram ensaiadas de acordo com a norma ASTM D 3163. Foram ensaiadas pelo menos 5 amostras para cada junta. Os ensaios foram feitos à temperatura ambiente, empregando-se uma máquina de ensaios de acionamento mecânico com capacidade de $100 \mathrm{kN}$. A velocidade de ensaio empregada foi de $1 \mathrm{~mm} / \mathrm{min}$. Após os ensaios, todos os corpos de prova foram examinados e apenas aqueles onde o defeito estava centrado, ou seja, não havia sido deslocado durante o processo de fabricação, foram validados. Assim, foram testados tantos corpos de prova quanto fossem necessários para obter pelo menos cinco resultados válidos.

As superfícies de fratura das juntas ensaiadas e a superfície do substrato na condição de como fabricado foram analisadas por microscopia eletrônica de varredura. As análises foram feitas usando-se o modo de operação com elétrons secundários e voltagem do feixe entre $15-20 \mathrm{kV}$. Como os corpos de prova examinados não eram condutores, foi feita a deposição de uma fina camada de ouro, de $\approx 3 \mathrm{~nm}$, sobre as superfícies observadas, para evitar acúmulo de carga nas amostras.

\section{Resultados e Discussão}

O valor medido para a rugosidade dos compósitos usados como aderentes foi de $11,00 \pm 0,85 \mu \mathrm{m}$. O pequeno coeficiente de variação obtido, $7,8 \%$, é uma indicação da uniformidade destes compósitos. Esta uniformidade do acabamento superficial, bem como a espessura constante do compósito, permitiram que a espessura da camada de adesivo fosse avaliada com precisão. As medidas das espessuras da camada de adesivo para os corpos de prova sem e com defeitos foram, respectivamente, de $0,09 \pm 0,02 \mathrm{~mm}$ e $0,21 \pm 0,04 \mathrm{~mm}$. A diferença observada é devida à espessura do Teflon, colocado como defeito, que é de $0,19 \mathrm{~mm}$. O resultado indica, entretanto, que o processo de fabricação empregado foi reprodutível, pois a espessura da camada de adesivo independeu do tamanho do defeito.

Na Tabela 1 está mostrada a variação da carga de ruptura das juntas em função do tamanho do defeito.
Pode-se observar que, embora haja um considerável espalhamento dos dados obtidos, a carga de ruptura de todas as juntas com defeitos é inferior ao valor da junta sem defeito. Este comportamento é válido mesmo para as juntas onde a área do defeito é bem pequena, por exemplo a junta D4 cuja área ocupada pelo defeito é de apenas $2 \%$ da área colada.

A Figura 4 mostra aspectos gerais da morfologia do compósito como fabricado, após a limpeza da superfície. Pode ser observada uma superfície irregular mostrando áreas ricas em resina $(\rightarrow)$ e áreas com fibras expostas $(\Rightarrow)$. Como mencionado anteriormente, um compósito com fibras de carbono unidirecionais foi usado como substrato. A aparência de tecido observada na Figura 4, é devida ao processo de fabricação do compósito, que é prensado com um tecido absorvedor ${ }^{[13]}$, o qual imprime $o$ aspecto de trançado superficialmente na resina matriz.

A morfologia geral da fratura das juntas está mostrada na Figura 5. Observa-se que a fratura foi não coesiva, isto é, a trinca se propaga ao longo da interface adesivo/substrato ${ }^{[18]}$. Nesta junta, apenas em alguns pontos isolados foram observadas pequenas regiões com fratura coesiva ${ }^{[3]}$. Também pode ser observado que a trinca muda de uma interface para a outra, formando um degrau na superfície de fratura. Este aspecto está de acordo com a variação de tensão na junta, conforme mostrado esquematicamente na Figura 3.

Outro aspecto importante observado na superfície de fratura, é a ausência de fibras expostas. Ou

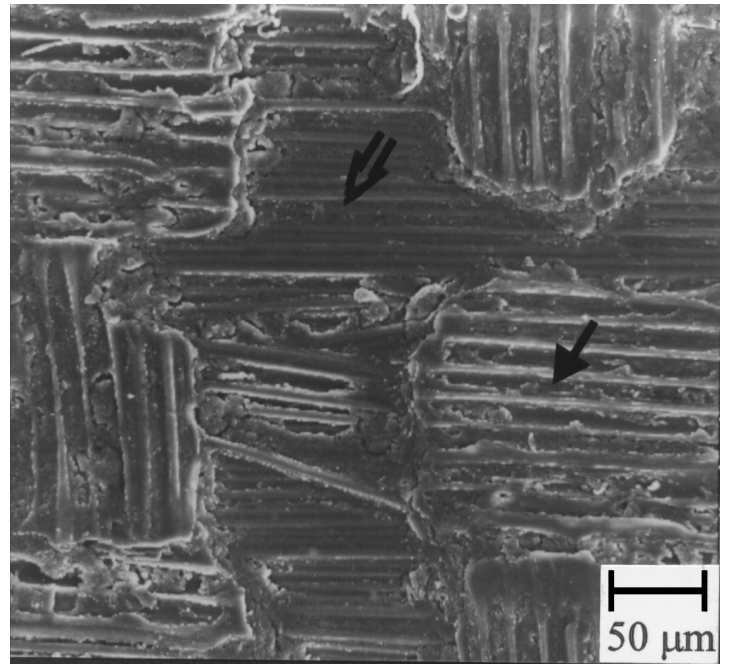

Figura 4. Aspectos gerais da morfologia do compósito na condição de como fabricado. 


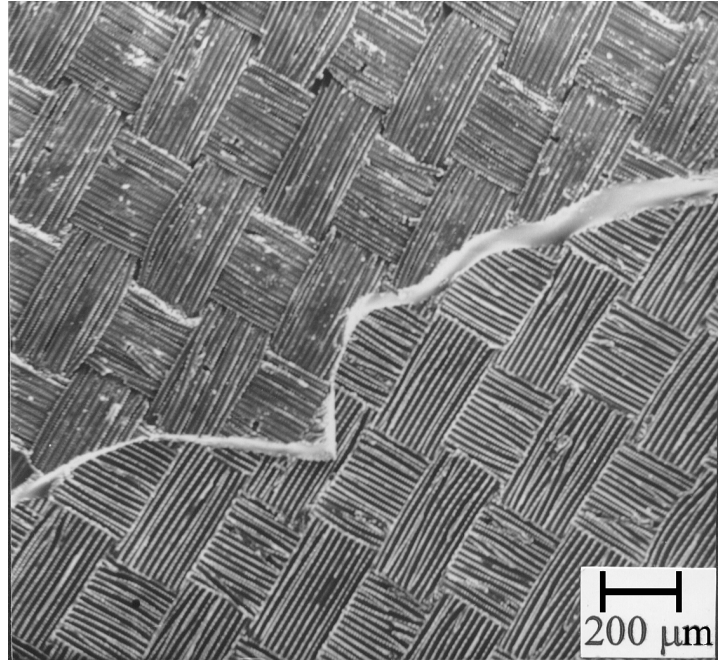

Figura 5. Morfologia geral da fratura decoesiva das juntas.

seja, o adesivo preencheu as imperfeições existentes no compósito, Figura 5 vs. Figura 4. Esta observação é importante, pois mostra que o adesivo teve um bom contato com o substrato, o que é encarado como requisito necessário para se obter uma boa união por $\operatorname{adesão}^{[15]}$.

A confirmação de que as estruturas superficiais, tipo tecido, observadas na Figura 4 não são fibras está mostrada na Figura 6a, onde se vê que estas estruturas são formadas pelo adesivo, quando este preenche os relevos deixados na superfície do compósito pelo tecido absorvedor. Na Figura 6b, é mostrado o degrau de fratura entre as duas interfaces adesivo/ substrato, e observa-se que a fratura atravessa toda a espessura do adesivo e, inclusive, as estruturas seme-

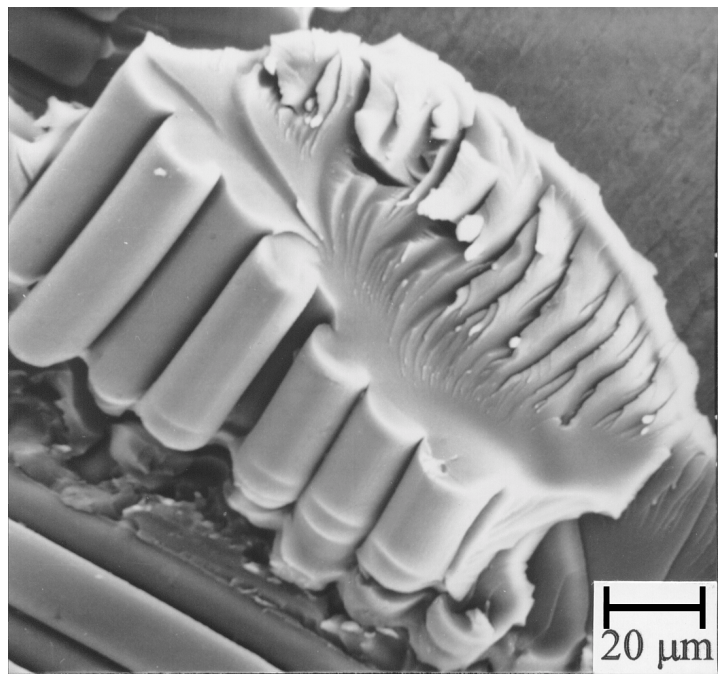

(a) lhantes a fibras. Além disso, os aspectos de fratura observados nestas estruturas são comuns aos aspectos fractográficos observados em polímeros ter$\operatorname{mofixos}^{[19,20]}$.

Analisando-se os dados mostrados na Tabela 1, pode-se notar que os grupos de corpos de prova $\mathrm{D} 4$, D6, D7 e D11 apresentam uma variação da carga de ruptura média aparentemente independente do tamanho do defeito. Entretanto, ao agrupá-los estatisticamente, isto é, calculando-se a média e o desvio padrão de dados agrupados, pode-se considerá-los como tendo uma mesma carga de ruptura média, pois apresentam um coeficiente de variação pequeno, $12 \%{ }^{[21]}$. O procedimento empregado na análise estatística destes dados está discutido detalhadamente em outro traba1 ho ${ }^{[4]}$. A partir dessa análise, pode-se determinar valores para a carga média e desvio padrão para os grupos de corpos de prova cujos resultados são estatisticamente diferentes. Estes resultados estão mostrados na Tabela 1.

Usando-se os dados corrigidos foi feita a análise do comportamento mecânico das juntas. Os valores da tensão cisalhante média estão mostrados na Tabela 2 e pode-se observar que são independentes do tamanho do defeito. De fato, analisados estatisticamente ${ }^{[4]}$, estes resultados podem ser considerados como idênticos, para um nível de significância de $95 \%$, e têm um valor médio de 8,24 MPa. Este resultado é importante, pois mostra que, para um tamanho de defeito de até $50 \%$ da área colada, a tensão cisalhante média atuando no adesivo é constante. Do

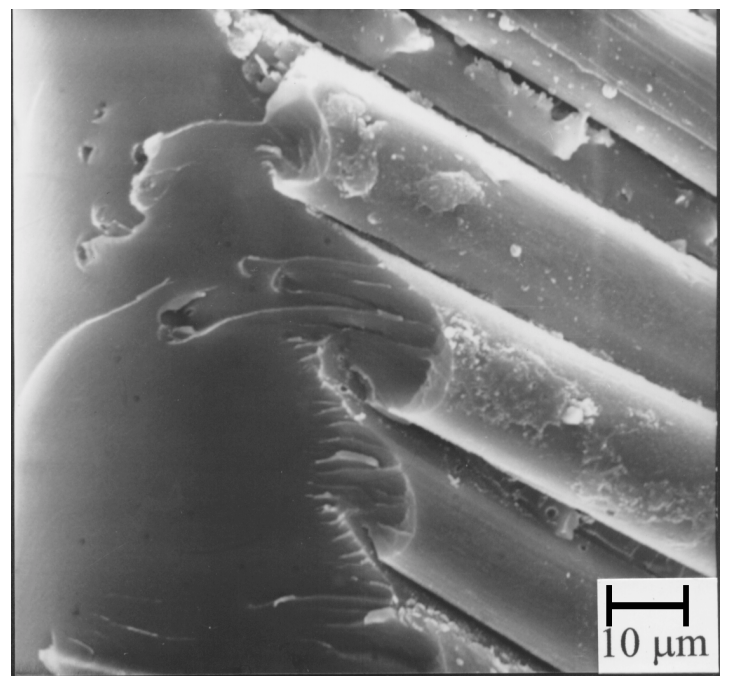

(b)

Figura 6. a)Detalhe das estruturas formadas pela matriz epoxi ao recobrir as irregularidades da superfície do compósito; b) Fratura ao longo do adesivo englobando as estruturas semelhantes a fibras. 
Tabela 2. Valores das tensões cizalhantes média e máxima

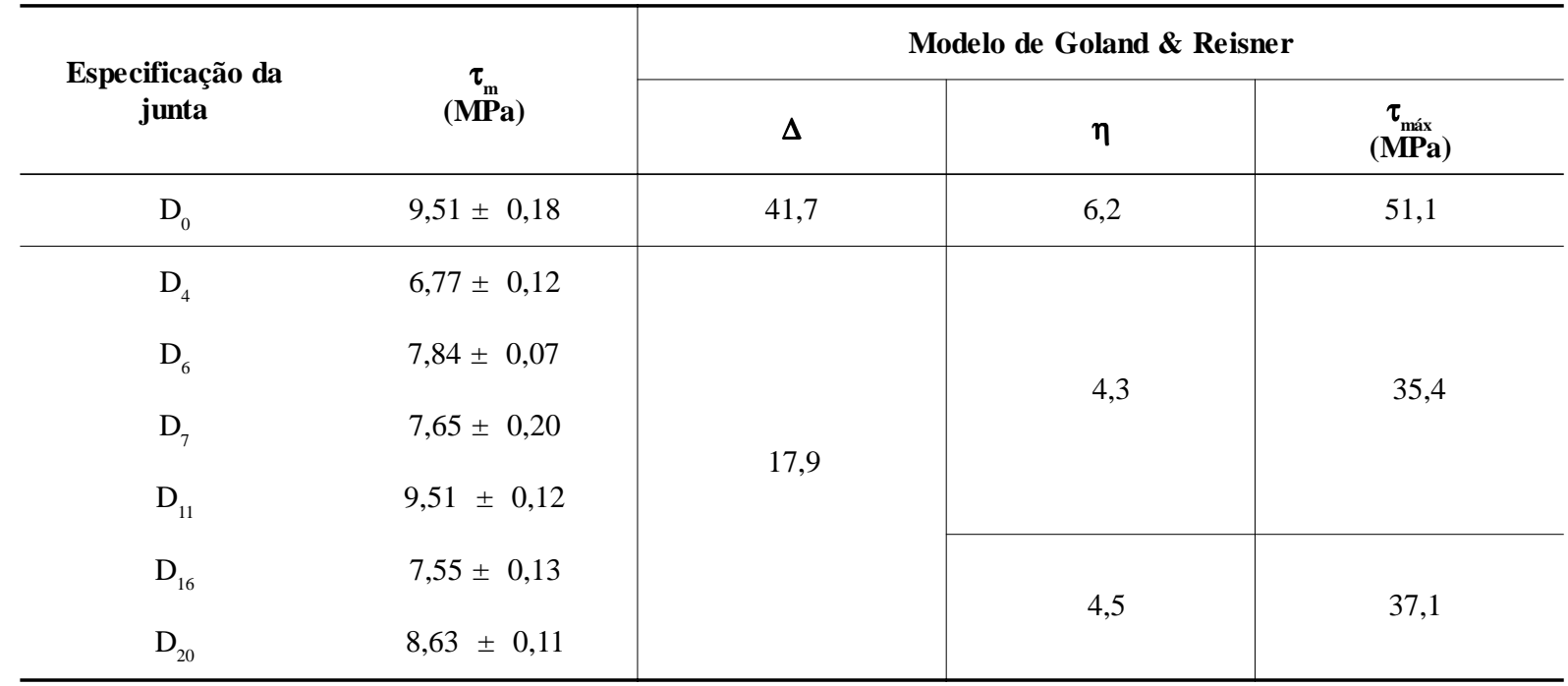

ponto de vista prático, este resultado é muito interessante, pois, se $\tau_{\mathrm{m}}$ é uma constante para uma dada configuração de junta, a carga de ruptura da junta pode ser predita conhecendo-se apenas a área real colada, ou seja, o tamanho do defeito presente.

A presença dos defeitos pode ser considerada como um modo de reduzir a rigidez da junta, de maneira semelhante ao que ocorre em compósitos laminados quando uma lâmina falha ${ }^{[22]}$. De fato, todas as juntas analisadas neste trabalho verificam a condição de juntas com camadas de adesivo flexíveis, e, portanto, contribuem significativamente para a distribuição de tensões na junta ${ }^{[7]}$. Assim sendo, foi feita uma análise da validade de se empregar para juntas com defeitos, os modelos usados para determinar as tensões em juntas sem defeitos.

Usando-se o modelo de Goland e Reissner ${ }^{[10]}$, a tensão cisalhante máxima atuando nas juntas pode ser obtida substituindo a equação (5) na expressão $\tau_{\text {máx }}=\eta \cdot \tau_{\mathrm{m}}$. Os valores obtidos estão mostrados na Tabela 2, tendo-se usado a tensão cisalhante média de 8,24 MPa para todas as juntas. Os resultados indicam que, aparentemente, $\eta$ diminui com a introdução de defeitos e, logo, o valor de máxima tensão cisalhante atuando na junta é reduzido. Estes resultados poderiam ser explicados se a rigidez da junta fosse reduzida pela introdução de defeitos.

A rigidez de uma junta do tipo sobreposta simples pode ser avaliada indiretamente através do parâmetro k, equação (4). Na Tabela 3 estão mostrados os valores de $\mathrm{k}$, calculados empregando-se dados característicos da junta estudada, ou seja: $v=0,30$;
$\mathrm{L}=25,4 \mathrm{~mm} ; \mathrm{s}=1,59 \mathrm{~mm} ; \mathrm{E}=116,52 \mathrm{GPa}$. Pode-se observar que o valor de $\mathrm{k}$ aumenta com o aumento do tamanho do defeito, o que indica que a rigidez da junta aumenta. Assim sendo, os resultados mostrados na Tabela 2 não indicam uma flexibilização da junta devido a introdução de defeitos. De fato, pode-se observar que o modelo sofre uma forte influência da espessura da camada de adesivo, conforme mostrado na Tabela 2 pelos valores de $\Delta$. A grande variação $\Delta$ influi nos valores do fator de concentração de tensões $\eta$ e, consequentemente, nos cálculos de $\tau_{\text {máx. }}$ Os resultados obtidos para $\tau_{\text {máx }}$ não podem assim ser diretamente comparados.

É interessante notar que embora a espessura das camadas de adesivo sejam pequenas em comparação com as espessuras do aderente, ou seja, possam ser consideradas como um filme, pequenas variações na espessura da camada de adesivo levam a grandes variações no cálculo de $\tau_{\text {máx }}$. Deste modo, os resultados obtidos mostram que o modelo de Goland e Reissner

Tabela 3. Variação do parâmetro k para cada grupo de corpos de prova

\begin{tabular}{ccc}
\hline Especificação & $\begin{array}{c}\text { Tensão Remota } \\
\text { (MPa) }\end{array}$ & $\mathbf{k}$ \\
\hline $\mathrm{D}_{0}$ & 151,8 & 0,52 \\
$\mathrm{D}_{4}$ & 106,79 & 0,56 \\
$\mathrm{D}_{6}$ & 120,42 & 0,55 \\
$\mathrm{D}_{7}$ & 115,32 & 0,55 \\
$\mathrm{D}_{11}$ & 128,85 & 0,54 \\
$\mathrm{D}_{16}$ & 84,53 & 0,59 \\
$\mathrm{D}_{20}$ & 69,33 & 0,61 \\
\hline
\end{tabular}

Polímeros: Ciência e Tecnologia, vol. 10, nº 3, p. 138-148, 2000 
Tabela 4. Variação de $\sigma_{\text {máx }}$ em função do tamanho dos defeitos

\begin{tabular}{|c|c|c|c|c|}
\hline Especificaçãa & $\gamma^{2}$ & $\begin{array}{c}\sigma_{\max } \\
(\mathbf{M P a})\end{array}$ & $\Delta^{1 / 2}$ & $\begin{array}{c}\sigma_{\max } \\
(\mathbf{M P a})^{*}\end{array}$ \\
\hline $\mathrm{D}_{0}$ & 0,09 & 3,53 & 6,46 & 55,31 \\
\hline $\begin{array}{l}\mathrm{D}_{4} \\
\mathrm{D}_{6} \\
\mathrm{D}_{7} \\
\mathrm{D}_{11}\end{array}$ & \multirow[t]{3}{*}{0,137} & 4,46 & \multirow[t]{3}{*}{4,23} & 38,34 \\
\hline $\mathrm{D}_{16}$ & & 3,43 & & 41,09 \\
\hline $\mathrm{D}_{20}$ & & 2,94 & & 43,05 \\
\hline
\end{tabular}

* cálculo pelo Modelo de Mylonas, equação (8)

só pode se adequar a análise da tensão cisalhante máxima atuando em juntas sobrepostas simples com defeitos se a espessura da camada de adesivo for mantida constante.

A equação simplificada dada pela equação (6) foi usada para o cálculo da máxima tensão normal atuando nas juntas. Os resultados obtidos estão mostrados na Tabela 4 , onde verifica-se que há novamente uma forte dependência da espessura da camada de adesivo, conforme mostram os valores de $\gamma^{2}$. Para as juntas $\mathrm{D}_{4}$ a $\mathrm{D}_{20}$, que têm a mesma espessura, os resultados mostram uma redução da $\sigma_{\text {máx }}$ com o aumento do defeito, indicando novamente a possibilidade de uma redução da rigidez efetiva da camada de adesivo. Deste modo, o momento fletor resultante é reduzido, pois a junta como um todo tende a se deformar mais facilmente para uma posição com quantidade mínima de energia deformação, conforme mostrado na Figura 2. Este comportamento tem como resultado reduzir as tensões normais atuantes na junta, o que corrobora os resultados obtidos.

A máxima tensão normal atuando na junta pode, também, ser analisada usando-se a equação (8). Pode-se observar o mesmo comportamento apresentado para os resultados dos valores de tensão cisalhante máxima, Tabela 2 . Ou seja, nesta equação tem-se também uma forte influência da espessura da camada de adesivo sobre os resultados obtidos. Os valores mostrados na Tabela 4 podem, assim, ser comparados apenas em relação aos grupos de amostras de mesma espessura.

Deve-se notar que os valores preditos pelos dois modelos diferem de uma ordem de grandeza. De fato, os valores obtidos pelo Modelo de Goland e
Reissner são baixos se compararmos os resultados com as tensões de ruptura do adesivo. Um valor típico para a tensão de ruptura sob tração uniaxial deste polímero é de $74 \mathrm{MPa}^{[23]}$. Assim, os valores preditos pela equação (8) podem ser encarados como mais adequados às observações experimentais. Valores nominais de tensão de ruptura inferiores aos observados quando o material é testado sob tração uniaxial são esperados, porque muitas vezes a ruptura de juntas unidas por adesão ocorre a partir da borda do sobreposto. Ou seja, na região onde existe concentração de tensão ${ }^{[7]}$.

O principal fato pelo qual a equação (8) se ajusta melhor às observações experimentais e os resultados por ela obtidos melhor se adaptam aos valores de tensão de ruptura esperados para resinas epoxi pode ser atribuído ao emprego de $\tau_{\mathrm{m}}$ e não de $\sigma$, tensão normal remota, como na equação (6). O uso da tensão remota não leva em consideração a presença de defeitos na junta, enquanto os valores de $\tau_{\mathrm{m}}$ estão diretamente relacionados com a área real colada.

Os resultados obtidos pela análise das juntas coladas com defeitos usando-se a equação do modelo de Hart-Smith, equação (9) estão mostrados na Tabela 5. Pode-se notar que neste modelo a variação da espessura é irrelevante, pois o termo (s+d) / 2 b mantém-se praticamente constante. Pode-se constatar ainda que, dentro da variação experimental, $\mathrm{M}_{\mathrm{e}}$ diminui com a introdução de defeitos. Assim, realmente, a rigidez efetiva da junta diminui quando se introduz defeitos, conforme já inferido pela análise dos resultados experimentais empregando-se os outros modelos.

Os valores calculados de $\sigma_{\text {máx }}$ são similares aos encontrados pelo Modelo de Mylonas, equação (8). 
Berry, N. G; d'Almeida, J . R. M. - Defeitos em juntas carbono-epoxi

Tabela 5 Resultados para a tensão normal atuando nas juntas coladas empregando-se o Modelo de Hart-Smith

\begin{tabular}{c|c|c|c|c}
\hline Especificação & $\begin{array}{c}\text { Carga } \\
(\mathbf{K g})\end{array}$ & $(\mathbf{s}+\mathbf{d}) / \mathbf{2 b}$ & $\begin{array}{c}\mathbf{M}_{\mathbf{e}} \\
(\mathbf{k g} / \mathbf{m m})\end{array}$ & $\begin{array}{c}\sigma_{\max } \\
(\mathbf{M P a})\end{array}$ \\
\hline $\mathrm{D}_{0}$ & 625,2 & 0,0331 & 10,7 & 63,2 \\
\hline $\mathrm{D}_{4}$ & & & 8,7 & 33,5 \\
$\mathrm{D}_{6}$ & & & 9,5 & 36,6 \\
$\mathrm{D}_{7}$ & 471,21 & \multirow{2}{*}{0,0354} & 9,1 & 38,9 \\
$\mathrm{D}_{11}$ & & & 10,1 & 38,9 \\
$\mathrm{D}_{16}$ & & & 7,2 & 27,7 \\
\hline $\mathrm{D}_{20}$ & 285,25 & & 6,2 & 23,7 \\
\hline
\end{tabular}

Como neste modelo não há a influência da diferença da espessura entre as camadas de adesivo para os corpos de prova com e sem defeito, todos os valores mostrados na Tabela 5 podem ser comparados. Estes resultados indicam que, realmente, o aumento do tamanho de defeito reduz a rigidez da junta e, portanto, reduz o momento fletor atuante. Assim, a máxima tensão normal a qual a junta está submetida é reduzida pela introdução de defeitos.

Deve-se ressaltar, entretanto, que embora $\sigma_{\text {máx }}$ diminua, logicamente, a performance da junta sem defeito é superior àquela com defeitos quando se analisa o comportamento das juntas em relação a carga de ruptura.

\section{Conclusões}

A colocação de um disco de Teflon para simular um defeito nas juntas unidas por adesão é satisfatória, pois não há adesão entre o Teflon e a resina utilizada como adesivo.

Para o sistema adesivo/aderente empregado e para o tipo de junta e carregamento analisado, a tensão cisalhante média é constante e função direta da área real colada. Ou seja, avaliando-se o tamanho do defeito, é possível prever a carga de ruptura da junta. Este resultado é considerado como bastante relevante do ponto de vista prático.

A partir dos resultados obtidos, pode-se concluir que o Modelo de Goland e Reissner pode ser adequado para analisar juntas sobrepostas simples com presença de defeitos de geometria circular e introduzidos no centro da junta. Deve-se observar, entretanto, que a espessura da camada de adesivo deve ser mantida constante para não influenciar nos resultados.

A introdução de defeitos centrais com geometria circular atua no sentido de reduzir a rigidez da camada de adesivo, favorecendo assim a deformação das juntas sobrepostas simples analisadas. Assim, embora a introdução de defeitos reduza a carga de ruptura da junta, os valores máximos das tensões atuantes nesta junta serão reduzidos.

Os valores preditos pelas equações de Mylonas e Hart-Smith podem ser encarados como os mais adequados às observações experimentais. Isto se deve ao uso de $\tau_{\mathrm{m}}$ ao invés de $\sigma$, pois os valores de $\tau_{\mathrm{m}}$ estão diretamente relacionados com a área real colada.

\section{Agradecimentos}

À PUC e à CAPES pelo suporte técnico e financeiro que tornaram viável este trabalho.

\section{Referências Bibliográficas}

1. Shields, J. - "Adhesive Bonding", Oxford University Press, Oxford (1974).

2. Bosma, M. e Oosterbroek, M. - Int.J.Adhesion and Adhesives, 14, p.93 (1994).

3. Berry, G. e d'Almeida, J.R.M. - Acta Microscopica , 6(A), p.304 (1997).

4. Berry,G. - “Avaliação da Influência de Defeitos sobre o Comportamento de Compósitos Unidos por Adesão", Dissertação de Mestrado, Departamento de Ciência dos Materiais e Metalurgia, PUC-RJ (1998).

5. De Bruyne, N.A and Houwink, R. - "Adhesion and Adhesives", Elsevier, New York (1951).

6. Pocius, A .V. - "Adhesion and Adhesives Technology, An Introduction", Hanser Publishers, Munich (1997). 
7. Kinloch, A.J. - "Adhesion and Adhesives Science and Technology", Chapman and Hall, London (1986).

8. Wang, C.H. e Rose, L.R.F. - Int. J. Adhesion and Adhesives, 17, p.17 (1996).

9. Volkersen, O. - Luftfahrtforsch, 15, p.41 (1938).

10. Goland, M. e Reissner, E. - J. Appl. Mechanics, 1, p.A17 (1944).

11. Mylonas, C. e De Bruyne, N. A. - "Adhesion and Adhesives" (eds N.A De Bruyne and R. Houwink) - Elsevier, Amsterdam, Cap.4, Static Problems, pp.91-143 (1951).

12. Hart-Smith, J.L. - "Analysis and Design of Advanced Composite Bonded joints"- NASA Report, no. CR2218, Washington-DC (1974).

13. Gomes Filho, A.L. e d'Almeida, J.R.M. - "Estágio Atual do Desenvolvimento de Materiais Conjugados na Divisão de Materiais do IPD” NT87/04, Centro Técnico Aeroespacial, São José dos Campos, SP (1987).

14. Naglis, M.M.M. e d'Almeida, J.R.M. - Polímeros: Ciência e Tecnologia, 8, p.54 (1998).

15. Allen, K.W. - Int. J. Adhesion and Adhesives, 13, p.67 (1993).
16. "Dow Epoxy Resins in Glass-Reinforced Plastics", Dow Química (1989).

17. Abdo, Z. and Aglan, H. - J. Mater. Sci. Letters, 15, p.469 (1996).

18. Garbassi, F., Morra, M. , Occhiello, E. - "Polymer Surfaces.From Physics to Technology", John Wiley \& Sons, Chichester, cap.10, pp.350-398 (1998).

19. Asp, L.E. , Berglund, L.A e Gudmumdson, P. Comp.Sci.\&Technology53, p.27 (1995).

20. Bandyopadhyay, S. - Materials Science and Engineering, A125, p.157 (1990).

21. Donaire, D. e Guerra, M.J. - "Estatística IndutivaTeoria e Aplicaçõs", Livraria Ciência e Tecnologia Editora, São Paulo, SP (1952).

22. Gibson, R.F. - "Principles of Composite Material Mechanics", McGraw-Hill, Singapore, cap.1, pp.1-33 (1994).

23. d'Almeida,J.R.M., Darwish,F.A.I. e Graça,M.L.A Rev.Bras.Ci.Mecânicas, 11, p.133 (1989).

Recebido: $29 / 02 / 00$

Aprovado: 29/08/00 